\title{
Difference in the Source of Anti-AQP4-IgG and Anti-MOG-IgG Antibodies in CSF in Patients With Neuromyelitis Optica Spectrum Disorder
}

\begin{abstract}
Tetsuya Akaishi, MD, PhD, Toshiyuki Takahashi, MD, PhD, Tatsuro Misu, MD, PhD, Kimihiko Kaneko, MD, PhD, Yoshiki Takai, MD, PhD, Shuhei Nishiyama, MD, PhD, Ryo Ogawa, MD, PhD, Juichi Fujimori, MD, PhD,

Tadashi Ishii, MD, PhD, Masashi Aoki, MD, PhD, Kazuo Fujihara, MD, PhD, and Ichiro Nakashima, MD, PhD
\end{abstract}

Neurology ${ }^{\circledR}$ 2021;97:e1-e12. doi:10.1212/WNL.0000000000012175

\section{Abstract}

\section{Objective}

To elucidate the differences in the source and in the level of intrathecal synthesis between anti-aquaporin-4 antibodies (AQP4-IgG) and anti-myelin oligodendrocyte glycoprotein antibodies (MOG-IgG).

\section{Methods}

Thirty-eight patients with MOG-IgG-associated disease and 36 with AQP4-IgG-positive neuromyelitis optica spectrum disorders (NMOSD) were studied for the antibody titers in the sera and CSF simultaneously collected in the acute attacks. The quotients between CSF and serum levels of albumin, total immunoglobulin G, and each disease-specific antibody were calculated. Intrathecal production level in each disease-specific antibody was evaluated by calculating the antibody index from these quotients.

\section{Results}

Eleven of the 38 patients with MOG-IgG were positive for the antibody only in the CSF, while no patient with AQP4-IgG showed CSF-restricted AQP4-IgG. Blood-brain barrier compromise as shown by raised albumin quotients was seen in $75.0 \%$ of MOG-IgG-positive cases and $43.8 \%$ of AQP4-IgG-positive cases. Moreover, MOG-IgG quotients were $>10$ times higher than AQP4-IgG quotients (effect size $r=0.659, p<0.0001)$. Elevated antibody index $(>4.0)$ was confirmed in 12 of 21 with MOG-IgG, whereas it was seen only in 1 of 16 with AQP4-IgG $(\varphi=$ $0.528, p<0.0001)$. The CSF MOG-IgG titers $(\rho=0.519, p=0.001)$ and antibody indexes for MOG-IgG $(\rho=0.472, p=0.036)$ correlated with the CSF cell counts but not with clinical disability.

\section{Conclusions}

Intrathecal production of MOG-IgG may occur more frequently than that of AQP4-IgG. This finding implies the different properties of B-cell trafficking and antibody production between MOG-IgG-associated disease and AQP4-IgG-positive NMOSD.

\author{
Correspondence \\ Dr. Akaishi \\ t-akaishi@med.tohoku.ac.jp
}

\section{RELATED ARTICLE}

Editorial

Intrathecal Production of MOG-IgG: Highlighting the Need for CSF Testing in Clinical Practice

Page 12 


\section{Glossary}

$\mathbf{A I}=$ antibody index; AQP4-IgG = anti-aquaporin-4 immunoglobulin G; CI = confidence interval; IgG = immunoglobulin G; IgG-spec = specific antibody; $\mathbf{I Q R}=$ interquartile range; MOG-IgG = anti-myelin oligodendrocyte glycoprotein immunoglobulin G; MOGAD = MOGAD; MS = multiple sclerosis; NMOSD = neuromyelitis optica spectrum disorder; $\mathrm{OCB}$ = oligoclonal band; $\mathrm{ON}$ = optic neuritis; $\mathrm{OR}=$ odds ratio.

Neuromyelitis optica spectrum disorder (NMOSD) is a demyelinating neurologic condition in the CNS. ${ }^{1,2}$ In contrast to multiple sclerosis (MS), which usually lacks disease-specific antibodies, NMOSD is characterized by the presence of specific antibodies such as anti-aquaporin-4 immunoglobulin G (AQP4-IgG) and anti-myelin oligodendrocyte glycoprotein immunoglobulin G (MOG-IgG).$^{3-5}$ Both patients with MOG-IgG-associated disease and those with AQP4IgG-positive NMOSD typically present with recurrent neurologic episodes represented by optic neuritis $(\mathrm{ON})$ and acute myelitis. ${ }^{4,6}$ Although these 2 conditions are likely to present similar clinical manifestations in the acute phase of attacks, the resulting neurologic sequelae are generally thought to be worse in AQP4-IgG-positive NMOSD, ${ }^{7-10}$ suggesting that these 2 disorders should be considered as independent disease entities with different approaches for relapse prevention. ${ }^{11-13}$ On the basis of the suggested differences in the clinical spectrum and eventual neurologic prognoses between patients with MOG-IgG and those with AQP4-IgG, neurologic conditions related to MOG-IgG have been considered separately from other conditions of NMOSD and regarded as an independent disease entity called the MOG-IgG-associated disease (MOGAD). ${ }^{14-17}$ Thereafter, the clinical need and rationale to discriminate between these 2 conditions have been vigorously discussed. ${ }^{18,19}$ Controversial topics include differences in properties of B-cell trafficking and antibody production site between these diseases. In this study, to evaluate the prevalence and clinical impact of intrathecal production of these disease-specific antibodies, we measured the titers of these antibodies in time-matched paired serum and CSF samples obtained in the acute phase of attacks.

\section{Methods}

\section{Patients and Disease Groups Based on Specific Antibody}

We initially recruited patients in our facility with acute neurologic episodes in whom time-matched paired serum and CSF MOG-IgG and AQP4-IgG titers were simultaneously evaluated during the acute phase of the neurologic episodes. The enrollment period for the patients treated in our facility (Tohoku University) was between 2006 and 2020. To increase the sample size, data of the patients treated in other facilities in Japan between 2019 and 2020 were additionally collected. Based on the results of the MOG-IgG and AQP4IgG titrations for their serum and CSF samples, patients were divided into the following 4 disease groups: MOGAD, AQP4IgG-positive NMOSD, MS without these antibodies, and other conditions (e.g., acute disseminated encephalomyelitis, neuro-Behçet disease, neuro-Sweet disease, cerebral infarction, idiopathic ON, and tumors). Patients with MOG-IgG in either serum or CSF were categorized as having MOGAD, and those with AQP4-IgG in either serum or CSF were categorized as having AQP4-IgG-positive NMOSD. Patients in the first 3 disease groups were enrolled in this study. Patients for whom time-matched paired serum and CSF samples were unavailable were not recruited in this study.

\section{Variables From Time-Matched Paired Serum and CSF Samples}

In each of the 3 enrolled disease groups, CSF cell count (mononuclear/polymorphonuclear), CSF protein level, presence of CSF-restricted oligoclonal bands (OCBs), and further CSF derivatives from time-matched paired serum and CSF samples were collected as follows: albumin quotient ( $\mathrm{Q}_{\mathrm{Alb}} ; \mathrm{CSF} /$ serum albumin ratio) and IgG quotient $\left(\mathrm{Q}_{\mathrm{IgG}-}\right.$ total; CSF/serum total IgG ratio), IgG index (i.e., a calculated value to estimate intrathecal total IgG synthesis), and antibody index (AI) for each specific antibody.

Titration of each specific antibody (IgG-spec: MOG-IgG or AQP4-IgG) was performed with a live cell-based assay as described in our previous reports. ${ }^{20,21}$ Screening of the serum samples to estimate seropositivity was performed at dilutions of 1:16 for AQP4-IgG and 1:128 for MOG-IgG; then, the antibody titers were calculated semiquantitatively using consecutive 2 -fold endpoint dilutions. Screening for CSF samples was performed without diluting the samples, and positive samples were further studied for antibody titers using the aforementioned semiquantitative serial dilution method. If MOG-IgG was positive only in the CSF, we additionally tested the serum of the patients at a dilution of 1:16 to exclude the presence of serum MOG-IgG with low titers between 1:16 and 1:64.

Using the time-matched paired serum and CSF samples in the acute phase, the following derivatives were comprehensively calculated from the equations described: $Q_{A l b}, Q_{I g G-t o t a l}$, $Q_{I g G-\text { spec }}, \operatorname{IgG}$ index, $Q_{\lim }(\operatorname{Ig} G), \mathrm{AI}$, and corrected AI.

$$
\begin{gathered}
Q_{A l b}=\frac{\text { Albumin level in CSF }[\mathrm{mg} / \mathrm{dl}]}{\text { Albumin level in serum }[\mathrm{mg} / \mathrm{dl}]} \\
Q_{I g G-\text { total }}=\frac{\operatorname{IgG} \text { level in } \operatorname{CSF}[\mathrm{mg} / \mathrm{dl}]}{\operatorname{IgG} \text { level in serum }[\mathrm{mg} / \mathrm{dl}]} \\
Q_{I g G-\text { spec }}=\frac{\text { Disease specific } \operatorname{IgG} \text { titer in CSF }[X]}{\text { Disease specific } \operatorname{IgG} \text { titer in serum }[X]}
\end{gathered}
$$


Table 1 Demographics and Laboratory Data for Different Disease Groups

\begin{tabular}{|c|c|c|c|c|c|}
\hline & MS & MOG-IgG (+) & AQP4-IgG (+) & Effect Size & $p$ Value \\
\hline No. & 83 & 38 & 36 & - & - \\
\hline Women, n (\%) & $63(75.9)$ & $23(60.5)$ & $33(91.7)$ & $0.363(\varphi)$ & 0.0024 \\
\hline Age at CSF study, $y^{a}$ & $30(25-39)$ & $23(8-44)$ & $52(46-63)$ & $0.552(r)$ & $<0.0001$ \\
\hline Optic neuritis at CSF study, n (\%) & $13 / 68(19.1)$ & $15 / 38(39.5)$ & 10/33 (30.3) & $0.096(\varphi)$ & 0.420 \\
\hline Acute myelitis at CSF study, $\mathrm{n}(\%)$ & $43 / 68(63.2)$ & 9/38 (23.7) & $22 / 33(66.7)$ & $0.432(\varphi)$ & 0.0003 \\
\hline Brain lesions at CSF study, $\mathrm{n}(\%)$ & $43 / 68(63.2)$ & $17 / 38(44.7)$ & $5 / 33(15.2)$ & $0.319(\varphi)$ & 0.0099 \\
\hline Times of previous neurologic episodes before CSF study & $0(0-0)$ & $0(0-0)$ & $0(0-1)$ & $0.083(r)$ & 0.475 \\
\hline \multicolumn{6}{|l|}{ CSF study results } \\
\hline CSF cell count, $n / \mu L^{a}$ & $3(1-7)$ & $15(4-39)$ & $8(3-40)$ & $0.029(r)$ & 0.810 \\
\hline Mononuclear cells, $n / \mu \mathrm{L}^{\mathrm{a}}$ & $3(1-7)$ & $14(4-36)$ & $7(3-32)$ & $0.049(r)$ & 0.683 \\
\hline PMNs, $n / \mu L^{a}$ & $0(0-0)$ & $0(0-2)$ & $1(0-2)$ & $0.092(r)$ & 0.447 \\
\hline CSF protein, $\mathrm{mg} / \mathrm{dL}^{\mathrm{a}}$ & $29(23-37)$ & $43(29-53)$ & $48(33-68)$ & $0.179(r)$ & 0.138 \\
\hline$Q_{A l b}, \times 10^{-3 a}$ & $4.5(3.5-5.7)$ & $5.8(4.1-7.9)$ & $6.2(3.9-9.7)$ & $0.016(r)$ & 0.924 \\
\hline$Q_{A l b}$ higher than age-specific reference range, $\mathbf{n}(\%)$ & $13 / 68(19.1)$ & $15 / 20(75.0)$ & $7 / 16(43.8)$ & $0.319(\varphi)$ & 0.087 \\
\hline$Q_{I g G-t \text { total }} \times 10^{-3 a}$ & $3.2(2.3-4.3)$ & $3.9(2.4-4.6)$ & $4.3(2.0-6.9)$ & $0.030(r)$ & 0.854 \\
\hline IgG index ${ }^{a}$ & $0.70(0.57-0.89)$ & $0.58(0.51-0.63)$ & $0.60(0.50-0.71)$ & $0.030(r)$ & 0.854 \\
\hline CSF-OCB, n (\%) & $59 / 80(73.8)$ & $12 / 27(44.4)$ & $3 / 13(23.1)$ & $0.207(\varphi)$ & 0.298 \\
\hline Serum IgG-spec titer, 1: $\mathrm{X}^{\mathrm{a}}$ & - & $128(0-1,024)$ & $8,192(1,024-32,768)$ & $0.581(r)$ & $<0.0001$ \\
\hline CSF IgG-spec titer, 1: $\mathrm{X}^{\mathrm{a}}$ & - & $8(4-16)$ & $16(4-32)$ & $0.124(r)$ & 0.286 \\
\hline$Q_{g G-s p e c}{ }^{a}$ & - & $0.063(0.010-\geq 1)$ & $0.0020(0.0010-0.0039)$ & $0.663(r)$ & $<0.0001$ \\
\hline \multicolumn{6}{|l|}{ Intrathecal MOG-IgG and AQP4-IgG syntheses } \\
\hline $\mathrm{Al}^{\mathrm{a}}$ & - & $4.6(2.7-73.2)$ & $0.65(0.15-1.03)$ & $0.659(r)$ & $<0.0001$ \\
\hline Corrected $\mathrm{Al}^{\mathrm{a}}$ & - & $4.6(3.2-56.3)$ & $0.65(0.21-1.03)$ & $0.659(r)$ & $<0.0001$ \\
\hline$A I \geq 1.5, n(\%)$ & - & $18 / 21(85.7)$ & $2 / 16(12.5)$ & $0.728(\varphi)$ & $<0.0001$ \\
\hline Corrected AI $\geq 1.5, n(\%)$ & - & $18 / 21(85.7)$ & $2 / 16(12.5)$ & $0.728(\varphi)$ & $<0.0001$ \\
\hline $\mathrm{Al}>4, \mathrm{n}(\%)$ & - & $12 / 21(57.1)$ & $1 / 16(6.3)$ & $0.528(\varphi)$ & 0.0016 \\
\hline Corrected Al >4, n (\%) & - & $12 / 21(57.1)$ & $1 / 16(6.3)$ & $0.528(\varphi)$ & 0.0016 \\
\hline
\end{tabular}

Abbreviations: AI = antibody index; AQP4-IgG = anti-aquaporin-4 antibodies; IgG = immunoglobulin G; IgG-spec = each specific antibody (i.e., MOG-IgG or AQP4-IgG); MOG-IgG = anti-myelin oligodendrocyte glycoprotein antibody; MS = multiple sclerosis; OCB = oligoclonal bands; $P M N=$ polymorphonuclear leukocyte; $Q_{A l b}=$ CSF/serum albumin quotient; $Q_{I g G-\text { spec }}=$ CSF/serum quotient of the titers for each specific antibody; $Q_{I g G-t o t a l}=$ CSF/serum IgG quotient. The shown effect sizes and $p$ values are for the comparisons between MOG-IgG-positive and AQP4-IgG-positive cases. Data from patients with MS were listed only for reference and were not included in the statistical comparisons. Effect sizes were described with $\varphi$ for the Fisher exact test or $r(=Z / \sqrt{N})$ for the MannWhitney $U$ test.

a Median and interquartile range (25th-75th percentile), followed by Mann-Whitney $U$ test.

${ }^{\mathrm{b}}$ Other comparisons of the prevalence are performed by the Fisher exact test.

$$
\operatorname{IgG} \text { index }=\frac{\mathrm{Q}_{\mathrm{Ig} G}}{\mathrm{Q}_{\mathrm{Alb}}}
$$

The upper reference limit of $Q_{A l b}$ is age dependent, which is usually calculated with the following equation ${ }^{22}$ :

$$
Q_{\text {Alb (upper limit })}=\frac{4+(\text { age } / 15)}{10^{3}}
$$

The values of $Q_{A l b}$ above this upper reference limit indicate blood-CSF barrier compromise.

A 2-dimensional scatterplot with $Q_{A l b}$ and $Q_{I g G}$ is called a Reibergram, which is useful for visually estimating the presence of intrathecal $\operatorname{IgG}$ synthesis and barrier dysfunction in the CNS. ${ }^{23}$ The range between the upper $\left(Q_{\text {lim }}\right)$ and lower hyperbolic discrimination line on the Reibergram includes 
$99 \%$ ( \pm 3 SDs) of previously investigated patients with miscellaneous conditions without intrathecal IgG synthesis. ${ }^{23,24}$ The upper line $\left(Q_{\text {lim }}\right)$ is defined by the following equation for each value of $Q_{A l b}$ :

$$
Q_{\lim }(I g G)=0.93 \sqrt{Q_{A l b}^{2}+6 \times 10^{-6}}-1.7 \times 10^{-3}
$$

A value above this line on the Reibergram indicates the presence of intrathecal IgG synthesis. ${ }^{25,26}$ This is useful for estimating the intrathecal synthesis for the total IgG level. After estimation of the intrathecal synthesis for each specific antibody, the value of the AI calculated by the following equation can be referenced:

$$
A I=\frac{Q_{I g G-\text { spec }}}{Q_{I g G-t o t a l}}
$$

The normal range of the $\mathrm{AI}$ is 0.6 to 1.3. Values of the $\mathrm{AI} \geq 1.5$ indicate intrathecal disease-specific IgG (i.e., MOG-IgG, AQP4-IgG) synthesis. ${ }^{27}$ As the above equation shows, the value of the $\mathrm{AI}$ is made up of 4 independent parameters (i.e., IgG-spec level in the CSF, serum IgG-spec level, total IgG level in the CSF, and total serum IgG level); thus, the value of the $\mathrm{AI}$ is relative and can increase after immunosuppressive treatments. ${ }^{27}$ The sensitivity of intrathecally synthesized specific antibodies can be increased by applying the concept of the corrected AI, which discriminates 2 cases as follows:

$$
\begin{gathered}
\text { Corrected } A I=\frac{Q_{I g G-\text { spec }}}{Q_{I g G-\text { total }}} \quad\left(\text { for } Q_{I g G \text {-total }}<Q_{\text {lim }}\right) \\
\text { Corrected } A I=\frac{Q_{I g G-\text { spec }}}{Q_{\text {lim }}} \quad\left(\text { for } Q_{\text {IgG-total }}>Q_{\text {lim }}\right)
\end{gathered}
$$

In this study, corrected AI was used as the AI values for MOGIgG and AQP4-IgG. If antibody titers instead of antibody concentration are used to calculate AI, as in the present study, the cutoff AI value of 4 is recommended for judging the presence of intrathecal antibody synthesis. ${ }^{23,28}$ Thus, the prevalence of patients with each specific antibody with $\mathrm{AI}>4$ was also evaluated.

\section{Statistical Analysis}

Distributions of quantitative variables were described as median (interquartile range $[\mathrm{IQR}]$; i.e., $25-75$ percentiles). Categorical variables were described as number and the prevalence (percent) in each disease group. Comparisons of quantitative variables between the 2 disease groups (i.e., MOG-IgG-positive and AQP4-IgG-positive groups) were performed with the Student $t$ test for variables with normal distributions and Mann-Whitney $U$ test for variables with nonnormal distributions. Comparisons of categorical variables between 2 groups were performed with the Fisher exact test. Correlations between 2 quantitative variables were evaluated with Spearman's rank correlation coefficient $(\rho)$. Correlations between binary variables and continuous variables were evaluated using point-biserial correlation coefficients $\left(r_{p b}\right)$. Test of no correlation was performed on the correlation coefficients $\left(\rho, r_{p b}\right)$ to judge the significance of correlations. For each statistical comparison, effect size with either of the following values was reported: $\varphi$, calculated as $\sqrt{\chi^{2} / N}$ ) or $r$ (calculated as $Z / \sqrt{N}$ ). To calculate the odds ratio (OR) with its $95 \%$ confidence interval $(\mathrm{CI})$ for the prevalence of intrathecal synthesis among MOG-IgG-positive cases, patients with AQP4-IgG were used as the reference group.

Statistical testing in this study was done at a 2-tailed $a$ level of 0.05 , and significance threshold correction based on the Bonferroni method was adopted as appropriate to adjust the statistical significance in multiple statistical comparisons. For the main outcomes (i.e., quotient of each specific antibody and $\mathrm{AI}$ for each specific antibody), a 2 -tailed $\alpha$ level of 0.05 was used as the threshold of statistical significance, whereas a 2 -tailed a level of 0.005 was adopted in other comparisons. Statistical analyses were performed with IBM SPSS Statistics 22.0 (IBM Corp, Armonk, NY).

\section{Standard Protocol Approvals, Registrations, and Patient Consents}

This study was approved by the Institutional Review Board of Tohoku University Graduate School of Medicine. Written informed consent was obtained from all enrolled patients.

\section{Data Availability}

All relevant data underlying the findings described in this study have been deposited in Dryad (doi.org/10.5061/dryad. wm37pvmmn).

\section{Results}

\section{Patients}

A total of 241 patients with acute neurologic episodes based on objective evidence of CNS lesions for whom time-matched paired serum and CSF samples were available were initially recruited in this study. Patients whose serum and CSF samples were obtained at different times with a time interval of 1 day or more (37 patients with MOG-IgG, 44 patients with AQP4-IgG, and 31 patients with MS) were not included.

According to the results of the titration tests for MOG-IgG and AQP4-IgG with the time-matched paired samples, the initially recruited 241 patients were further divided into the following 4 disease groups: 38 patients with MOGAD, 36 patients with AQP4-IgG-positive NMOSD, 83 patients with MS, and 84 patients with other conditions. Patients in the first 3 disease groups were enrolled in this study. Eleven of the 38 MOG-IgG-positive patients (28.9\%) were seronegative with only CSF-restricted MOG-IgG. In these 11 patients with CSF-restricted MOG-IgG, 4 were with isolated ON, 2 were with acute myelitis, 3 were with cerebral lesions, and the remaining 2 were with mixed distributions (i.e., ON, myelitis, and cerebral lesions). The remaining 27 patients were MOGIgG seropositive with $(\mathrm{n}=24)$ or without $(\mathrm{n}=3)$ MOG-IgG in the CSF. All the $36 \mathrm{AQP} 4-\mathrm{IgG}$-positive patients were 
Figure 1 Simultaneous Titers of Specific Antibodies (MOG-IgG, AQP4-IgG) in the Serum and CSF

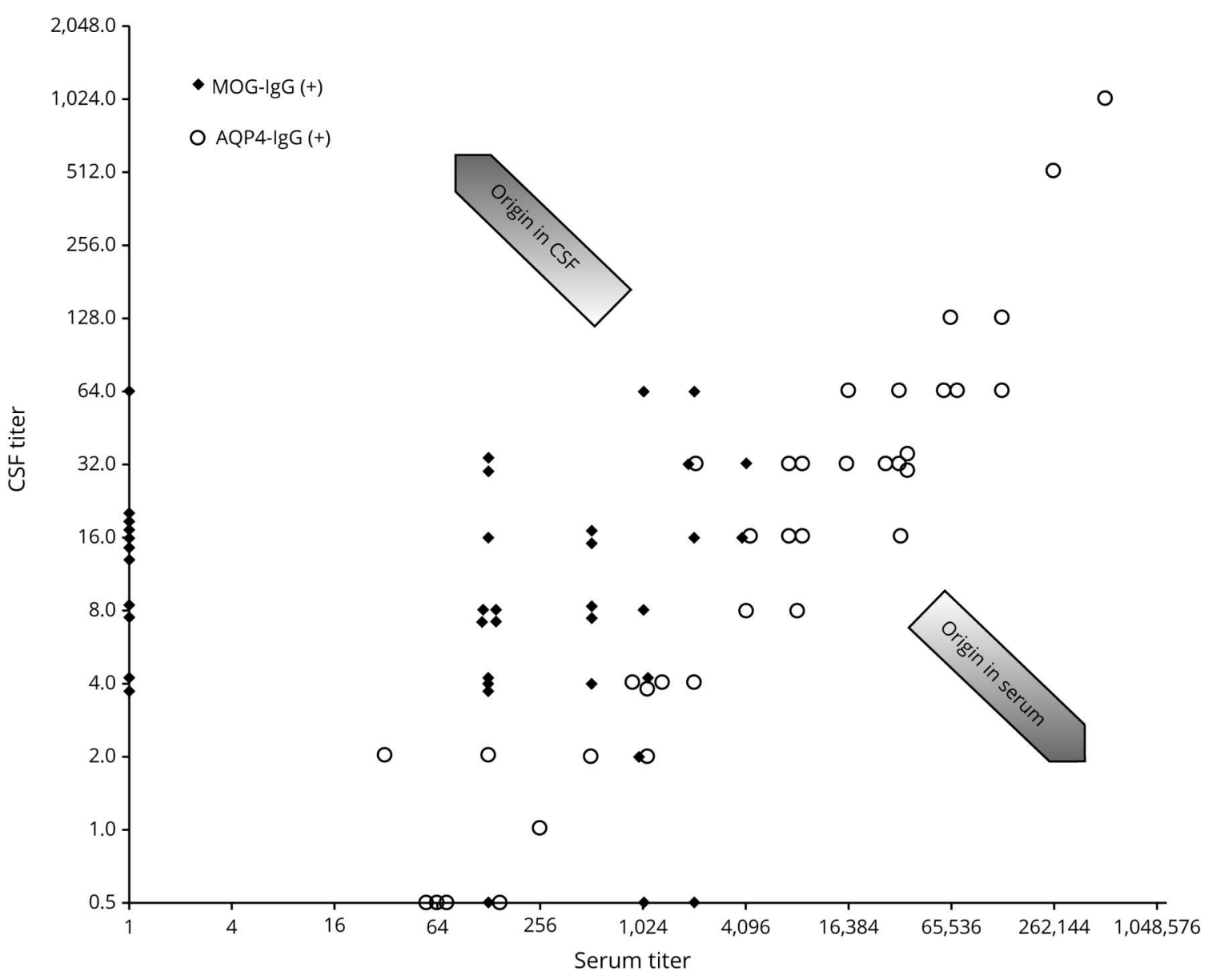

Anti-myelin oligodendrocyte glycoprotein antibody (MOG-IgG) showed higher quotients (i.e., ratio of CSF/serum levels) than anti-aquaporin-4 antibodies (AQP4-IgG) regardless of the serum titer levels. This shows that a larger proportion of specific antibody in the CSF originates intrathecally in MOG-IgG compared to AQP4-IgG.

seropositive with $(\mathrm{n}=32)$ or without $(\mathrm{n}=4)$ AQP4-IgG in the CSF.

The paired serum and CSF samples in the acute phase of attacks before starting acute treatments were available for all patients with MOG-IgG, but 4 of the 38 patients ( 2 with CSFrestricted MOG-IgG, 2 with serum MOG-IgG) had already undergone relapse prevention treatment (low-dose oral prednisolone) before sample collection. Furthermore, the paired samples in the acute phase of attacks were available for all patients with AQP4-IgG, but 1 of the 36 patients was enrolled just after the initiation of high-dose IV steroid therapy as acute treatment. Four of the 36 patients with AQP4IgG had already undergone relapse prevention treatment (3 with low-dose oral prednisolone and 1 with oral prednisolone and methotrexate) before sample collection. Twenty-eight of the 38 MOG-IgG-positive patients were analyzed at onset, whereas the remaining 10 were analyzed during relapses. Twenty-five of the 36 AQP4-IgG-positive patients were analyzed at onset, whereas the other 11 were analyzed during relapses.

\section{Demographics and Laboratory Data}

The demographics and measured laboratory data in each disease group are listed in the upper third of table 1. Female rate was $74.7 \%$ in the MS group, $60.5 \%$ in the MOGAD group, and $91.7 \%$ in the AQP4-IgG-positive NMOSD group. The age distribution was significantly higher in AQP4IgG-positive NMOSD group than in the other 2 disease groups. Among 21 of the 38 MOG-IgG-positive patients (55.3\%) and 16 of the 36 AQP4-IgG-positive patients (44.4\%), $Q_{I g G-t o t a l}$ values were obtained at the same time as the titration for each specific antibody, enabling the calculation of AI. Furthermore, the $Q_{\text {Alb }}$ was obtained for 20 of the 38 MOG-IgG-positive patients and 16 of the 36 AQP4IgG-positive patients. The IgG index was obtained for 23 of the 38 MOG-IgG-positive patients and 28 of the 36 AQP4IgG-positive patients. The level of CSF cell counts was slightly higher in cases with MOG-IgG or AQP4-IgG than in patients with MS. Pleocytosis with CSF white blood cell count $>5 / \mu \mathrm{L}$ was observed in 22 of 35 (62.9\%) patients with MOGIgG and in 21 of 35 (60.0\%) patients with AQP4-IgG. The protein level in the CSF was also higher in cases with MOG- 


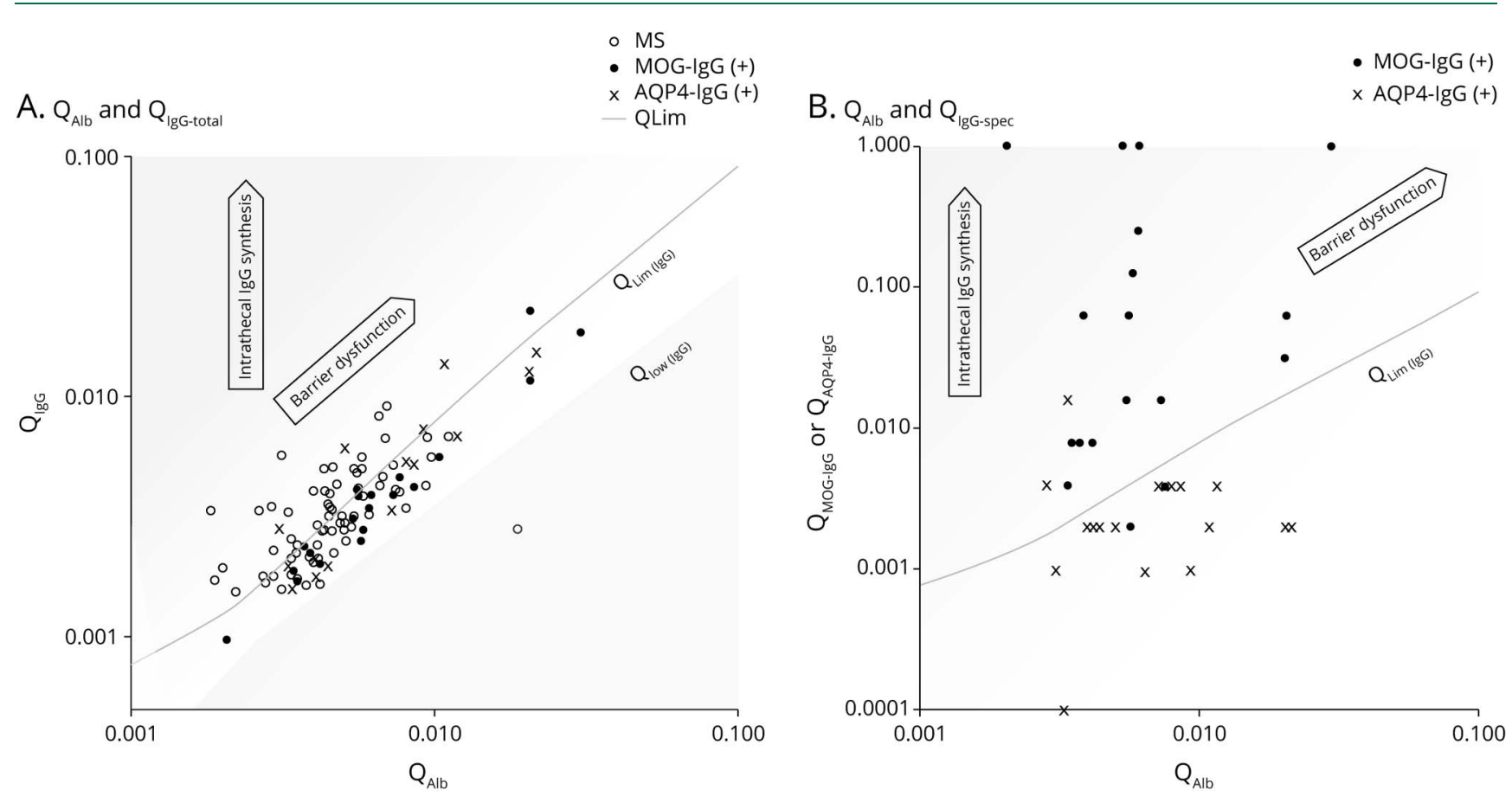

(A) Scatterplots with albumin quotient $\left(Q_{A l b}\right)$ and immunoglobulin G quotient ( $\left.Q_{I g G-t o t a l}\right)$ on the Reibergram. Plots in patients with anti-myelin oligodendrocyte glycoprotein antibody (MOG-IgG) or anti-aquaporin-4 antibodies (AQP4-IgG) distribute below and along the upper line of the distribution of patients without intrathecal IgG synthesis on the Reibergram $\left(Q_{L i m}\right)$ line, whereas many of them were with raised $Q_{A l b}$. (B) Scatterplots with $Q_{A l b}$ and quotient for each specific antibody (i.e., $\left.Q_{M O G-I g G}, Q_{A Q P 4-I g G}\right)\left(Q_{I g G-s p e c}\right)$ on the Reibergram. Plots in patients with MOG-IgG distribute above the $Q_{L i m}$ line and not along the line. MS $=$ multiple sclerosis.

$\operatorname{IgG}$ (Cohen $d=0.776$, Student $t$ test, $p=0.0004)$ or AQP4$\operatorname{IgG}$ (Cohen $d=1.121, p<0.0001)$ than in cases with MS. The blood- and CSF-derived quotients are listed in the middle of table 1. Both $Q_{\text {Alb }}$ and $Q_{g G \text {-total }}$ were lowest in patients with MS, while the derived IgG index was highest in these patients. The distribution of $Q_{g G-s p e c}$ in cases with MOG-IgG $\left(Q_{M O G-I g G}\right)$ was significantly higher than that in cases with AQP4-IgG ( $\left.Q_{A Q P 4-I g G}\right)$. To visually confirm this difference, a scatterplot with simultaneous serum and CSF titers for each specific IgG (i.e., MOG-IgG and AQP4-IgG) is shown in figure 1. Eleven of the 38 patients with MOG-IgG showed positive results for the antibody in the CSF alone and negative results for that in the serum. None of the patients with serum AQP4-IgG showed positive results for the antibody in the CSF alone. The prevalence of OCB was not different between patients with CSF-restricted MOG-IgG and other MOG-IgG seropositive patients ( 4 of 9 [44.4\%] vs 8 of 18 [44.4\%], $\varphi=$ 0.000 , Fisher exact test, $p=1.000)$. The number of cases with respective clinical phenotypes of MOGAD in OCB-positive and -negative groups was as follows: 4 with $\mathrm{ON}, 2$ with acute myelitis, and 6 with brain involvement in 12 OCB-positive cases, and 7 with ON, 3 with acute myelitis, and 5 with brain involvement in 15 OCB-negative cases.

\section{Intrathecal IgG Synthesis and Barrier Dysfunction by Disease Group}

Next, $Q_{A l b}$ and $Q_{I g G-t o t a l}$ for each patient were plotted on the Reibergram for all the disease groups, as shown in figure $2 \mathrm{~A}$.
Most patients with MOG-IgG or AQP4-IgG were distributed below the hyperbolic curve of $Q_{\lim }(\operatorname{Ig} G)$, which was different from the distribution of the patients with MS. A raised $Q_{A l b}$ value above the age-specific reference range (i.e., suggestive of blood-CSF barrier compromise) was confirmed in 15 of 20 MOG-IgG-positive patients, whereas raised $Q_{\text {Alb }}$ was confirmed in 7 of the 16 AQP4-IgG-positive patients $(75.0 \%$ vs $43.8 \%, \varphi=0.319$, Fisher exact test, $p=$ $0.0874)$. The prevalence of raised $Q_{\text {Alb }}$ in patients with MOG-IgG was significantly higher than the $19.1 \%$ prevalence in patients with MS $(\varphi=0.503, p<0.0001)$, whereas the difference between AQP4-IgG-positive patients and patients with MS narrowly failed to reach statistical significance $(\varphi=0.227, p=0.0517)$. If we use the cutoff value of 0.60 as the upper reference limit of $\operatorname{IgG}$ index, 9 of the 23 MOG-IgG-positive patients and 15 of 28 AQP4IgG-positive patients showed elevated IgG index $(39.1 \%$ vs $53.6 \%, \varphi=0.144, p=0.4004)$. The prevalence of increased IgG index in patients with MOG-IgG was significantly lower than that in 54 of $78(69.2 \%)$ patients with MS ( $\varphi=0.261, p=0.0136)$ but not in those with AQP4-IgG ( $\varphi$ $=0.145, \chi^{2}$ test, $\left.p=0.1359\right)$.

Meanwhile, as shown in the previous section, $Q_{g G-s p e c}$ for MOG-IgG and AQP4-IgG showed a completely different distribution. On this basis, scatterplots with $Q_{A l b}$ and $Q_{I g G-s p e c}$ by disease group (i.e., MOG-IgG associated, AQP4-IgG positive) were also plotted on the Reibergram, as shown in figure $2 \mathrm{~B}$. 

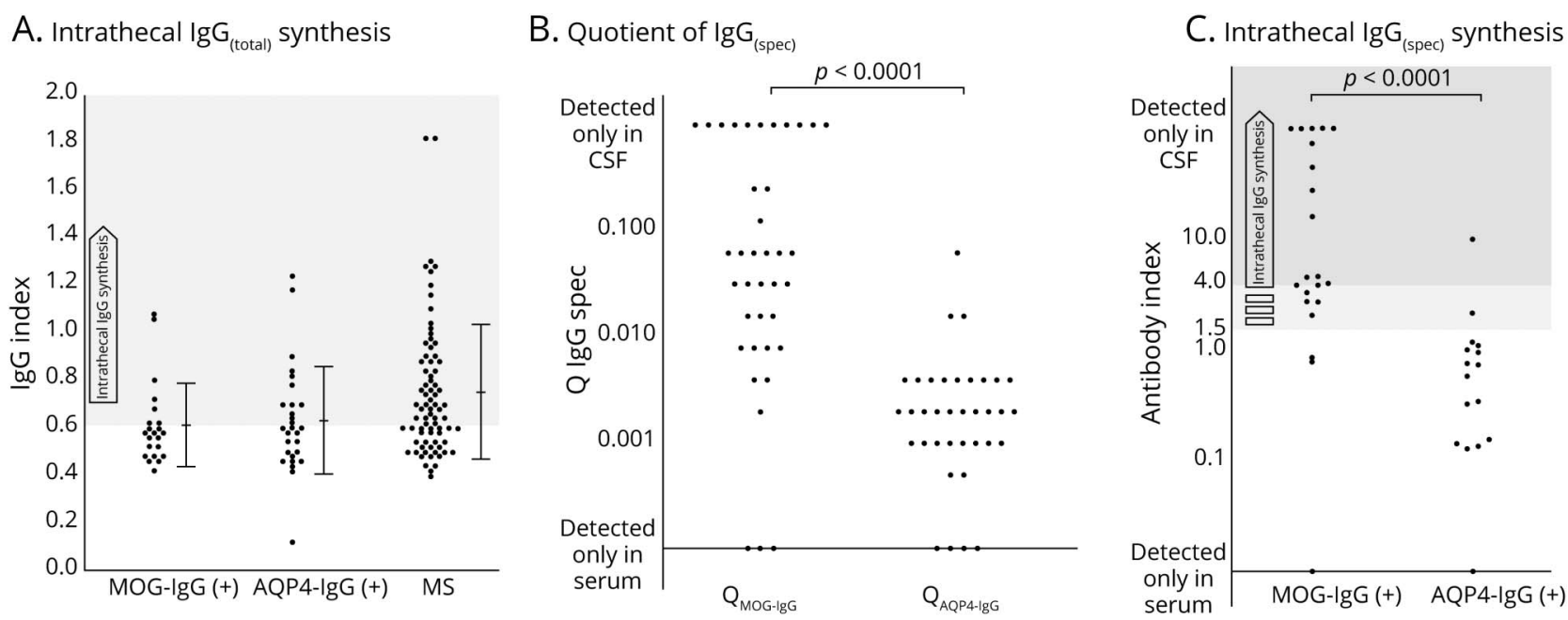

Grouped scatterplots by disease groups for (A) immunoglobulin $G(\operatorname{IgG})$ index, (B) quotient for each specific antibody (i.e., $\left.Q_{\text {MoG-IgG }}, Q_{A Q P 4-I g G}\right)\left(Q_{I g G-s p e c}\right)$ and (C) antibody index (Al) are shown. IgG index reflects the intrathecal total IgG production, whereas $Q_{I g G-s p e c}$ and Al represent the intrathecal production of each specific antibody. AQP4-IgG = anti-aquaporin-4 antibodies; MS = multiple sclerosis; MOG-IgG = anti-myelin oligodendrocyte glycoprotein antibody.

\section{Intrathecal Synthesis of MOG-IgG and AQP4-IgG} With the achieved values of $Q_{I g G-t o t a l}$ and $Q_{I g G-s p e c}$, the AI was calculated for each of the MOG-IgG-positive and AQP4IgG-positive groups. Although the distribution of $Q_{A l b}$ and $Q_{I g G-t o t a l}$ in the patients with MOG-IgG did not surpass the $Q_{\text {lim }}$ line (suggesting the absence of intrathecal IgG synthesis), the quotient of the specific MOG-IgG showed totally different results. Grouped scatterplots for the IgG index, $Q_{g g-s p e c}$, and $\mathrm{AI}$ in each disease group are shown in figure 3. Both the AI values (effect size $r=0.659$, Mann-Whitney $U$ test, $p<$ $0.0001)$ and the rate of cases with an $\mathrm{AI} \geq 1.5$ ( $\varphi=0.728$, Fisher exact test, $p<0.0001$ ) were significantly higher in the MOG-IgG-positive patients than in the AQP4-IgG-positive patients. The calculated unadjusted $\mathrm{OR}$ on taking an $\mathrm{AI}$ value $\geq 1.5$ among MOG-IgG-positive patients compared to AQP4IgG-positive patients as reference was 42.00 (95\% CI 6.15-286.68). The AI value was still significant even after we excluded the 5 cases with CSF-restricted MOG-IgG (effect size $r=0.603, p=0.0006$ ). When an AI value $>4$ was adopted for judging the presence of intrathecal synthesis, the prevalence of cases with $\mathrm{AI}>4$ was still significantly higher in MOG-IgG-positive patients ( $57.1 \%$ vs $6.3 \%, \varphi=0.528, p=$ $0.0016)$ with the calculated OR of $20.00 \quad(95 \%$ CI 2.21-180.69).

\section{Association Between Al for MOG-IgG and Clinical Manifestation}

To identify the background factor leading to the production of an elevated AI in patients with MOG-IgG, we checked the relationship between the corrected AI values (i.e., level of intrathecal synthesis for each specific antibody) and other variables (e.g., clinical manifestation and laboratory data). The Spearman correlation coefficient $(\rho)$ for each pair of the variables in each disease group is listed in table 2. The CSF level of MOG-IgG significantly correlated with the CSF cell count $(\rho=0.519, p=0.0014)$. Meanwhile, the correlation between CSF level of AQP4-IgG and the CSF cell count was weaker $(\rho=0.311, p=0.0691)$. The clinical manifestation of encephalitis was not associated with an elevated AI for MOG$\operatorname{IgG}\left(r_{p b}=0.047, p=0.7813\right)$. In detail, the median (IQR) of the AI for MOG-IgG among those with encephalitis was 4.6 (IQR 2.7- $\geq 100 ; n=9)$ and that among those without encephalitis was 4.3 (IQR 3.2-39.2; $n=12$ ), suggesting the same level of intrathecal MOG-IgG synthesis regardless of the clinical manifestation of encephalitis. The median of the AI for MOG-IgG among those with ON was 73.2 (IQR 4.3- $\geq 100$; n $=7$ ), which was slightly higher than the 3.9 (IQR $2.7-25.0 ; \mathrm{n}$ = 14) among those without ON but did not reach statistical significance (effect size $r=0.361$, Mann-Whitney $U$ test, $p=$ 0.0985).

\section{After Exclusion of Cases With Relapse Prevention and With CSF-Restricted MOG-IgG}

Last, because the evaluated cohort included some patients with relapse prevention treatment at the CSF study, comparison of AI values between MOG-IgG-positive and AQP4IgG-positive cases was additionally performed after the exclusion of those treated with relapse prevention or with acute treatments at the lumbar punctures. Furthermore, to exclude the bias from the heterogeneity of the cohort with MOG-IgG seropositive cases and others with CSF-restricted MOG-IgG, we further excluded the MOG-IgG seronegative cases with CSF-restricted MOG-IgG. Consequently, AI values from 15 MOG-IgG seropositive cases and 12 AQP4-IgG seropositive cases were eligible for further analyses. Scatterplots of $Q_{g G-t o t a l}$ and $Q_{g G-s p e c}$ among these serologically homogeneous cohorts are shown in figure 4 . The serum titers of each disease-specific antibody were 1:128 or higher in all patients 
Table 2 Correlation Coefficients Between the Al and Other Variables

\begin{tabular}{|c|c|c|c|c|}
\hline & \multicolumn{2}{|l|}{ MOG-IgG } & \multicolumn{2}{|l|}{ AQP4-IgG } \\
\hline & CSF MOG-IgG Titer $(n=38)$ & Corrected MOG-AI $(n=21)$ & CSF AQP4-IgG Titer $(n=36)$ & Corrected AQP4-AI $(n=16)$ \\
\hline Men $^{a}$ & $0.079(p=0.64)$ & $-0.045(p=0.85)$ & $-0.061(p=0.73)$ & $-0.123(p=0.65)$ \\
\hline Age at CSF study ${ }^{b}$ & $-0.081(p=0.63)$ & $-0.060(p=0.80)$ & $0.109(p=0.53)$ & $-0.041(p=0.88)$ \\
\hline Disease duration at CSF study ${ }^{b}$ & $-0.202(p=0.22)$ & $0.249(p=0.28)$ & $-0.373(p=0.033)$ & $0.413(p=0.13)$ \\
\hline No. of attacks before CSF study ${ }^{b}$ & $0.018(p=0.92)$ & $0.341(p=0.13)$ & $-0.289(p=0.088)$ & $0.424(p=0.10)$ \\
\hline Moderate to severe cases $^{a, c}$ & $-0.209(p=0.27)$ & $0.008(p=0.98)$ & $0.251(p=0.23)$ & $-0.320(p=0.27)$ \\
\hline \multicolumn{5}{|c|}{ Type of clinical manifestation at CSF study ${ }^{a}$} \\
\hline Brain involvement & $0.047(p=0.78)$ & $0.222(p=0.33)$ & $-0.147(p=0.414)$ & $-0.151(p=0.61)-$ \\
\hline ON & $0.008(p=0.96)$ & $0.351(p=0.12)$ & $0.169(p=0.35)$ & $0.525(p=0.054)$ \\
\hline Acute myelitis & $-0.040(p=0.81)$ & $-0.029(p=0.90)$ & $0.179(p=0.32)$ & $0.190(p=0.51)$ \\
\hline \multicolumn{5}{|l|}{ Laboratory datab } \\
\hline White blood cell count & $0.216(p=0.30)$ & $0.194(p=0.46)$ & $0.430(p=0.041)$ & $-0.051(p=0.86)$ \\
\hline CSF cell count (total) & $0.519(p=0.001)$ & $0.472(p=0.036)$ & $0.311(p=0.069)$ & $-0.499(p=0.049)$ \\
\hline Mononuclear cells & $0.489(p=0.003)$ & $0.474(p=0.035)$ & $0.316(p=0.069)$ & $-0.502(p=0.047)$ \\
\hline PMNs & $0.490(p=0.003)$ & $0.341(p=0.14)$ & $0.282(p=0.11)$ & $-0.271(p=0.31)$ \\
\hline Serum total protein level & $-0.001(p=1.00)$ & $0.157(p=0.56)$ & $-0.193(p=0.44)$ & $0.603(p=0.050)$ \\
\hline CSF protein level & $0.183(p=0.30)$ & $-0.114(p=0.63)$ & $0.401(p=0.017)$ & $-0.429(p=0.097)$ \\
\hline \multicolumn{5}{|c|}{$\begin{array}{l}\text { Abbreviations: } \mathrm{Al}=\text { antibody index; } \mathrm{AQP4}-\mathrm{IgG}=\text { anti-aquaporin-4 antibodies; MOG-IgG = anti-myelin oligodendrocyte glycoprotein antibody; ON }=\text { optic } \\
\text { neuritis; PMN = polymorphonuclear leukocyte. } \\
\text { The shown } p \text { values aside from each correlation coefficient are the results of the test of no correlation. Values of } p<0.005 \text { were regarded to be statistically } \\
\text { significant by adopting the Bonferroni correction for multiple statistical comparisons. } \\
\text { a The shown correlation coefficients are point-biserial correlation coefficients }\left(r_{p b}\right) \text {. } \\
\text { b The shown correlation coefficients are the Spearman rank correlation coefficients ( } \rho \text { ). } \\
\text { c Moderate to severe clinical severity was judged by the Expanded Disability Status Scale score (cutoff }>3.0 \text { ) at CSF study in } 30 \text { MOG-IgG-positive patients and } \\
25 \text { AQP4-IgG-positive patients. }\end{array}$} \\
\hline
\end{tabular}

except for AQP4-IgG in 1 patient (serum AQP4-IgG titer 1:64, CSF AQP4-IgG negative). The distribution of AI value was still significantly higher in MOG-IgG seropositive cases than in AQP4-IgG seropositive cases (effect size $r=0.653$, MannWhitney $U$ test, $p=0.0007$ ). The rate of $\mathrm{AI}>4$ was also still significantly higher in MOG-IgG seropositive cases than in AQP4- $\operatorname{IgG}$ seropositive cases ( 6 of 15 vs 0 of $12, \varphi=0.478$, Fisher exact test, $p=0.0200)$.

\section{Discussion}

In the present study, we evaluated simultaneously acquired serum and CSF samples to compare the concurrent serum and CSF titers for each specific antibody (i.e., MOG-IgG and AQP4-IgG). Thereafter, we evaluated the correlations between the levels of intrathecal production in these diseasespecific antibodies and patient demographics or clinical data. The observed general CSF parameters in the present cohorts with MOG-IgG or AQP4-IgG (e.g., CSF white blood cell count, CSF protein) were similar to those reported in previous studies from other countries. ${ }^{29}$ As an exception, the prevalence of CSF-restricted OCB in MOG-IgG-positive patients in the present cohort (44.4\%) was higher than that previously reported $(5 \%-20 \%) .{ }^{29-31}$ This may be partly attributed to the relatively high rate of MOG-IgG-positive patients with cerebral of brainstem lesions in the present cohort. ${ }^{30}$ In our cohort, there were 77 other patients with MOGAD who were excluded from this study due to unavailability of paired serum and CSF samples in the acute phase of attacks. Among them, 57 had ON, 9 had acute myelitis, and 20 had brain involvement, which is different from the proportion of the clinical phenotypes of the patients with MOGAD enrolled in the present study. This may have contributed to a relatively high OCB-positive rate in the patients with MOGAD included in this study. The calculated value of the IgG index, a marker of intrathecal total-IgG synthesis that is elevated in most patients with MS, ${ }^{32,33}$ was not elevated in either of the patients with MOG-IgG or AQP4-IgG. Meanwhile, the prevalence of raised $Q_{A l b}$, suggesting disrupted blood-CSF barrier function, was significantly higher in MOGIgG-positive patients than that in patients with MS. These facts clearly imply that patients with MOG-IgG and those with AQP4-IgG have distinct properties in blood-brain/ 


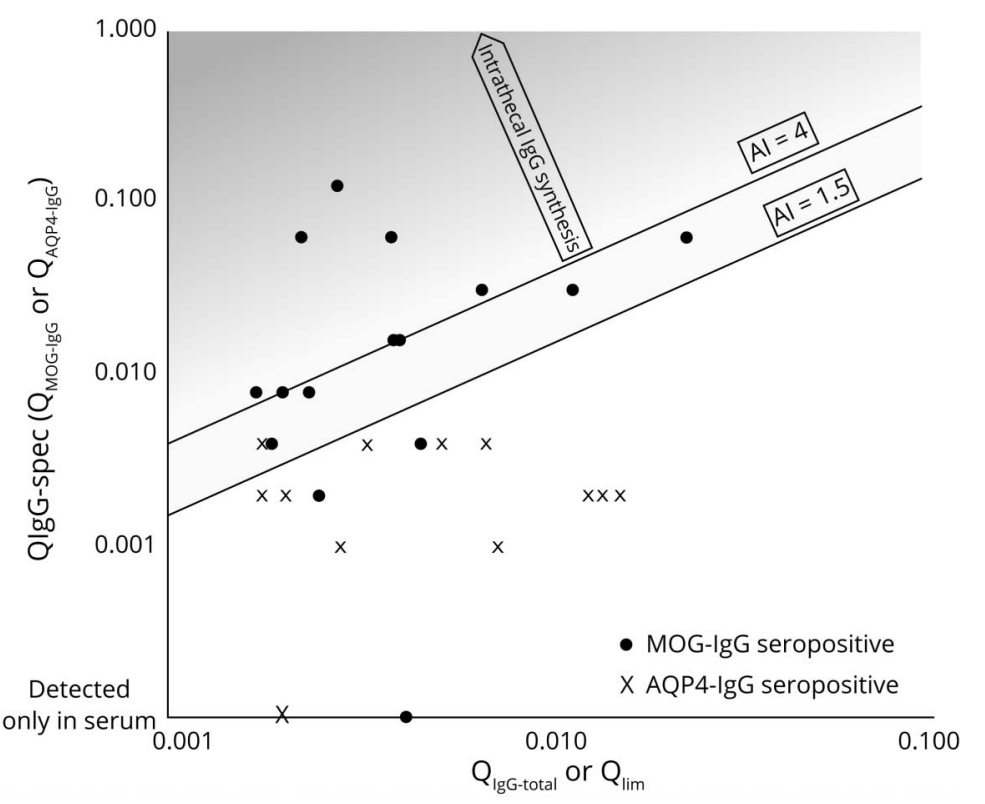

Scatterplots of antibody index (Al) after the exclusion of patients with relapse prevention therapy or with CSF-restricted anti-myelin oligodendrocyte glycoprotein antibody (MOG-IgG) are shown. Both the distribution of Al value and the prevalence of AI value $\geq 1.5$ or $>4$ were still significantly higher in the MOG-IgG seropositive patients than in the anti-aquaporin-4 antibodies (AQP4-IgG) seropositive patients. Diagonal solid lines represent the Al values of 1.5 and 4.0. IgG = immunoglobulin $\mathrm{G} ; \mathrm{Q}_{\mathrm{Ig} G \text {-spec }}=$ quotient for each specific antibody (i.e., $Q_{M O G-I g G}, Q_{A O P 4-I g G}$ ); $Q_{\lim }=$ upper line of the distribution of patients without intrathecal IgG synthesis on the Reibergram.
blood-CSF barrier permeability and intrathecal total IgG production from patients with MS, compatible with the general conception that the clinical manifestations in MOGAD largely overlap with those in AQP4-IgG-positive NMOSD rather than with those in MS. ${ }^{2,3}$ Meanwhile, the results of the present study demonstrated that the levels of intrathecal production for each disease-specific antibody were largely different between MOG-IgG and AQP4-IgG. Both the calculated $Q_{I g G-s p e c}$ (i.e., $Q_{M O G-I g G}, Q_{A Q P 4-I g G}$ ) and AI suggested that most of the MOG-IgG in the CSF is intrathecally produced by the CSF plasmablasts migrated from the peripheral blood, whereas most of AQP4-IgG in the CSF is extrathecally produced and passively transferred from the blood into the CSF.

The pathogenicity of disease-specific antibodies (MOG-IgG, AQP4-IgG) in the CNS has generally been thought to be exerted after they are passively transferred from the peripheral blood into the CNS or CSF through an open blood-brain barrier or blood-CSF barrier. ${ }^{34}$ One of the reasons for this general conception is the absence of an elevated IgG index and the reduced prevalence of OCB in patients with MOGIgG or with AQP4-IgG compared to patients with MS. ${ }^{35,36}$ Although AQP4-IgG in the CSF has been shown to be largely derived from tissue-resident (e.g., bone marrow) or circulating peripheral AQP4-IgG-producing plasmablasts, ${ }^{28}$ some of these peripheral AQP4-IgG-producing plasmablasts migrate into the CNS or the intrathecal space and are involved in intrathecal AQP4-IgG production. ${ }^{34,37}$ In fact, AQP4-specific CSF plasmablast was cloned from the CSF lymphocytes of the AQP4-IgG-positive patients. $^{38}$ Meanwhile, intrathecal MOG-IgG production is still controversial. An elevated rate of intrathecal MOG-IgG production was reported to be present in some of the patients diagnosed with MS on the basis of the titers measured with the ELISA method. ${ }^{39}$ Another study reported that most of the MOG-IgG-positive patients do not show intrathecal MOG-IgG production according to the titers measured with a cell-based assay, indicating a predominant peripheral origin of CSF MOG-IgG. ${ }^{40}$ More recently, several case series with acute neurologic episodes accompanied by CSF-restricted MOG-IgG have been reported. ${ }^{41,42}$ Whether the patients with CSF-restricted MOG-IgG should be managed separately from other MOG-IgG seropositive patients has been a recent topic of discussion. In this study, the measured CSF MOG-IgG titer was $\approx 10$ to 100 times higher than that estimated when assuming that all MOG-IgG in the CSF is passively transferred from the blood without intrathecal synthesis. Furthermore, the CSF titer and calculated AI for MOG-IgG significantly correlated with the CSF white blood cell count, supporting the hypothesis that these CSF white blood cells intrathecally produce MOG-IgG that comprises most of the CSF MOG-IgG. Notably, 5 of the 9 patients with CSF-restricted MOG-IgG who were studied for OCB were negative for the presence of OCB. This fact implies that the intrathecal synthesis of MOG-IgG cannot always be detectable by the elevated IgG index or OCB positivity. Such intrathecal MOG-IgG synthesis can be evaluated safely only by calculating $Q_{M O G-I g G}$ and $\mathrm{AI}$ in each patient. A possible explanation for the observed difference in the origin sites of MOG-IgG and AQP4-IgG in the CSF may be the different distributions of molecular expression between MOG and AQP4 proteins. The molecular expression of MOG is generally limited to the nervous system, ${ }^{43,44}$ whereas that of AQP4 ranges broadly across the human body and is not limited to the nervous system. ${ }^{45,46}$ The expression of MOG protein limited to the nervous system would result in the 
antigen-specific B-cell differentiation and maturation into MOG-IgG-producing plasmablasts within the CNS or CSF. This theory is further supported by the observed correlation between the calculated MOG-AI and CSF white blood cell count. It should be noted that this study does not deny the presence and role of intrathecal AQP4-IgG production. As described above, AQP4-IgG-producing plasmablasts in the CSF have been already identified and cloned in a few previous studies. ${ }^{34,37,38}$ Another previous study demonstrated correlations between the CSF AQP4-IgG titer and CSF levels of some proinflammatory cytokines such as interleukin- $1 \beta$ and interleukin-6. ${ }^{47}$ Furthermore, this study is not enough to conclude the clinical impact of intrathecal disease-specific IgG production because the subsequent clinical course and neurologic disturbance level in the chronic phase after acute treatments were evaluated in fewer than half of the enrolled patients. As shown in table 2, neither MOG-AI nor AQP4-AI showed a positive correlation with the concurrent neurologic disability level in the CSF study, a finding that was compatible with a previous study that reported that the estimated intrathecal MOG-IgG production level did not correlate with clinical disability or radiographic outcome measures. ${ }^{39}$ Further studies are warranted to evaluate the clinical impact of intrathecal MOG-IgG and AQP4-IgG production on the clinical severity or prognosis of patients with these antibodies.

This study has several limitations. First, all enrolled patients were of Asian ethnicity, and these results are not generalized due to the study being restricted to Asians. Second, the titers for MOG-IgG and AQP4-IgG were measured semiquantitatively with 2-fold endpoint dilutions. Consequently, the measured titers may have some extent of errors with 2-fold at most, and the calculated $Q_{g G-s p e c}$ may have errors with 4-fold at most. However, because this possible bias is applied to all measured cases, the conclusions are not significantly affected by such errors derived from the measurement system. Moreover, we applied the AI cutoff value at 4 , not 1.5 , in this study that used titers, not antibody concentrations. Third, possible influence of the antigen sink effect by MOG and AQP4 proteins on cellular membrane to the measured titers for each disease-specific antibody was not considered in this study. It has been known that therapeutic monoclonal antibodies can be deprived and eliminated faster in low dose by unbound targets on the cellular surface, especially when the target antigens are internalizing receptors. ${ }^{48,49}$ While the CSF AQP4-IgG titer may have decreased due to this sink model, the model was unlikely to have resulted in the elevated MOG-AI level. Consequently, the overall results of this study were not significantly biased by the possible antigen sink effect; however, the exact decrement in CSF AQP4IgG titer by the effect remains unknown. Another limitation could be the small sample size, as is evident from the wide CIs for all unadjusted ORs. Last, the samples from most of the evaluated patients were collected at the first clinical episode. The observed findings might be different if we measure the same data among the patients with relapses at the second or later clinical episodes.
Among patients with NMOSD with either AQP4-IgG or MOG-IgG, a larger proportion of MOG-IgG than AQP4-IgG is intrathecally produced in the CSF. Such differences in proportions of intrathecal origin between the antibodies in the CSF may reflect the different properties of B-cell trafficking and antibody production between MOGAD and AQP4-IgG-positive NMOSD.

\section{Study Funding}

This study is not industry sponsored. This work was supported by MHLW Program grant 20FC1030 and JSPS KAKENHI grant 20K07892.

\section{Disclosure}

T. Akaishi, T. Takahashi, T. Misu, K. Kaneko, Y. Takai, S. Nishiyama, R. Ogawa, J. Fujimori, T. Ishii, and M. Aoki report no disclosures. K. Fujihara received speaker honoraria and travel funding from Bayer, Biogen Japan, Eisai, Mitsubishi Tanabe, Novartis, Astellas, Takeda, Asahi Kasei Medical, Daiichi Sankyo, and Nihon Pharmaceutical and received research support from Bayer, Biogen, Asahi Kasei Medical, the Chemo-Sero-Therapeutic Research Institute, Teva, Mitsubishi Tanabe Pharma, Teijin, Chugai, Ono, Nihon Pharmaceutical, and Genzyme. I. Nakashima received speaker honoraria and travel funding from Mitsubishi Tanabe Pharma, Biogen Japan, and Novartis Pharmaceuticals and received research support from LSI Medience Corp. Go to Neurology.org/ $\mathrm{N}$ for full disclosures.

\section{Publication History}

Received by Neurology November 13, 2020. Accepted in final form March 22, 2021.

Appendix Authors

\begin{tabular}{lll}
\hline Name & Location & Contribution \\
\hline $\begin{array}{l}\text { Tetsuya } \\
\text { Akaishi, MD, } \\
\text { PhD }\end{array}$ & $\begin{array}{l}\text { Department of Neurology, } \\
\text { Toho University, Japan }\end{array}$ & $\begin{array}{l}\text { Drafting the manuscript, } \\
\text { study concept and design, } \\
\text { analysis and interpretation } \\
\text { of data, acquisition of data, } \\
\text { statistical analysis }\end{array}$ \\
\hline
\end{tabular}

Toshiyuki Department of Neurology, Study concept and design,

Toshiyuki Department of Neurology, Takahashi, Tohoku University, Japan analysis and interpretation MD, PhD of data, acquisition of data, statistical analysis, revising the manuscript

\begin{tabular}{lll}
\hline Tatsuro & Department of Neurology, & Analysis and interpretation \\
Misu, MD, & Tohoku University, Japan & $\begin{array}{l}\text { of data, acquisition of data, } \\
\text { statistical analysis, revising } \\
\text { PhD }\end{array}$ \\
& & $\begin{array}{l}\text { the manuscript, study } \\
\text { supervision }\end{array}$
\end{tabular}

Kimihiko Department of Neurology, Analysis and interpretation Kaneko, MD, Tohoku University, Japan of data, acquisition of data, PhD statistical analysis, revising the manuscript

Yoshiki Department of Neurology, Analysis and interpretation Takai, MD, Tohoku University, Japan of data, acquisition of data PhD statistical analysis, revising the manuscript 
Appendix (continued)

\begin{tabular}{lll}
\hline Name & Location & Contribution \\
\hline $\begin{array}{l}\text { Shuhei } \\
\text { Nishiyama, } \\
\text { MD, PhD }\end{array}$ & $\begin{array}{l}\text { Department of Neurology, } \\
\text { Tohoku University, Japan }\end{array}$ & $\begin{array}{l}\text { Analysis and interpretation } \\
\text { of data, acquisition of data, } \\
\text { statistical analysis, revising } \\
\text { the manuscript }\end{array}$
\end{tabular}

Ryo Ogawa, Department of Neurology,

MD, PhD Tohoku University, Japan

Analysis and interpretation of data, acquisition of data, statistical analysis, revising the manuscript

\begin{tabular}{ll}
\hline Juichi & Department of Neurology, \\
Fujimori, & Tohoku Medical and \\
MD, PhD & Pharmaceutical University,
\end{tabular}
Japan

Analysis and interpretation of data, acquisition of data, statistical analysis, revising the manuscript

\begin{tabular}{lll}
\hline Tadashi & Department of Education & Analysis and interpretation \\
Ishii, MD, & $\begin{array}{l}\text { and Support for Regional } \\
\text { of data, statistical analysis, }\end{array}$ \\
PhD & Medicine, Tohoku University & revising the manuscript \\
& Hospital, Japan &
\end{tabular}

\begin{tabular}{ll}
\hline Masashi & Department of Neurology, \\
Aoki, MD, & Tohoku University, Japan
\end{tabular}

Revising the manuscript, study concept and design, interpretation of data, acquisition of data, study supervision

\begin{tabular}{|c|c|c|}
\hline $\begin{array}{l}\text { Kazuo } \\
\text { Fujihara, } \\
\text { MD, PhD }\end{array}$ & $\begin{array}{l}\text { Department of Multiple } \\
\text { Sclerosis Therapeutics, } \\
\text { Fukushima Medical } \\
\text { University, Japan }\end{array}$ & $\begin{array}{l}\text { Revising the manuscript, } \\
\text { study concept and design, } \\
\text { interpretation of data, } \\
\text { acquisition of data, study } \\
\text { supervision }\end{array}$ \\
\hline $\begin{array}{l}\text { Ichiro } \\
\text { Nakashima, } \\
\text { MD, PhD }\end{array}$ & $\begin{array}{l}\text { Department of Neurology, } \\
\text { Tohoku Medical and } \\
\text { Pharmaceutical University, } \\
\text { Japan }\end{array}$ & $\begin{array}{l}\text { Revising the manuscript, } \\
\text { study concept and design, } \\
\text { interpretation of data, } \\
\text { acquisition of data, study } \\
\text { supervision }\end{array}$ \\
\hline
\end{tabular}

\section{References}

1. Pittock SJ, Lucchinetti CF. Neuromyelitis optica and the evolving spectrum of autoimmune aquaporin-4 channelopathies: a decade later. Ann NY Acad Sci. 2016; 1366(1):20-39.

2. Hor JY, Asgari N, Nakashima I, et al. Epidemiology of neuromyelitis optica spectrum disorder and its prevalence and incidence worldwide. Front Neurol. 2020;11:501.

3. Wingerchuk DM, Banwell B, Bennett JL, et al. International consensus diagnostic criteria for neuromyelitis optica spectrum disorders. Neurology. 2015;85(2):177-189.

4. Jarius S, Paul F, Aktas O, et al. MOG encephalomyelitis: international recommendations on diagnosis and antibody testing. J Neuroinflammation. 2018;15(1):134.

5. Lennon VA, Kryzer TJ, Pittock SJ, Verkman AS, Hinson SR. IgG marker of opticspinal multiple sclerosis binds to the aquaporin-4 water channel. J Exp Med. 2005; 202(4):473-477.

6. Pandit L, Mustafa S, Nakashima I, Takahashi T, Kaneko K. MOG-IgG-associated disease has a stereotypical clinical course, asymptomatic visual impairment and good treatment response. Mult Scler J Exp Transl Clin. 2018;4(3):2055217318787829.

7. Akaishi T, Nakashima I, Takeshita T, et al. Different etiologies and prognoses of optic neuritis in demyelinating diseases. J Neuroimmunol. 2016;299:152-157.

8. Mariano R, Messina S, Kumar K, Kuker W, Leite MI, Palace J. Comparison of clinical outcomes of transverse myelitis among adults with myelin oligodendrocyte glycoprotein antibody vs aquaporin-4 antibody disease. JAMA Netw Open. 2019;2(10):e1912732.

9. Song H, Zhou H, Yang M, et al. Different characteristics of aquaporin-4 and myelin oligodendrocyte glycoprotein antibody-seropositive male optic neuritis in China. J Ophthalmol. 2019;2019:4015075.

10. Liu $\mathrm{H}$, Zhou $\mathrm{H}$, Wang J, et al. The prevalence and prognostic value of myelin oligodendrocyte glycoprotein antibody in adult optic neuritis. J Neurol Sci. 2019;396:225-231.

11. Kitley J, Waters P, Woodhall M, et al. Neuromyelitis optica spectrum disorders with aquaporin-4 and myelin-oligodendrocyte glycoprotein antibodies: a comparative study. JAMA Neurol. 2014;71(3):276-283.

12. Höftberger R, Sepulveda M, Armangue T, et al. Antibodies to MOG and AQP4 in adults with neuromyelitis optica and suspected limited forms of the disease. Mult Scler. 2015;21(7):866-874.

13. Borisow N, Mori M, Kuwabara S, Scheel M, Paul F. Diagnosis and treatment of NMO spectrum disorder and MOG-encephalomyelitis. Front Neurol. 2018;9:888.

14. Juryńczyk M, Jacob A, Fujihara K, Palace J. Myelin oligodendrocyte glycoprotein (MOG) antibody-associated disease: practical considerations. Pract Neurol. 2019; 19(3):187-195.
15. Kim H, Lee EJ, Kim S, et al. Serum biomarkers in myelin oligodendrocyte glycoprotein antibody-associated disease. Neurol Neuroimmunol Neuroinflamm. 2020;7(3):e708.

16. Tajfirouz DA, Bhatti MT, Chen JJ. Clinical characteristics and treatment of MOGIgG-associated optic neuritis. Curr Neurol Neurosci Rep. 2019;19(12):100.

17. Parrotta E, Kister I. The expanding clinical spectrum of myelin oligodendrocyte glycoprotein (MOG) antibody associated disease in children and adults. Front Neurol. 2020;11:960.

18. Sato DK, Callegaro D, Lana-Peixoto MA, et al. Distinction between MOG antibodypositive and AQP4 antibody-positive NMO spectrum disorders. Neurology. 2014; 82(6):474-481.

19. Cobo-Calvo A, Sepúlveda M, Rollot F, et al. Evaluation of treatment response in adults with relapsing MOG-Ab-associated disease. J Nuroinflammation. 2019;16(1):134.

20. Takahashi T, Fujihara K, Nakashima I, et al. Establishment of a new sensitive assay for anti-human aquaporin-4 antibody in neuromyelitis optica. Tohoku J Exp Med. 2006; 210(4):307-313

21. Takahashi T, Fujihara K, Nakashima I, et al. Anti-aquaporin-4 antibody is involved in the pathogenesis of NMO: a study on antibody titre. Brain. 2007;130(pt 5): 1235-1243.

22. Reiber H. Cerebrospinal fluid: physiology, analysis and interpretation of protein patterns for diagnosis of neurological diseases. Mult Scler. 1998;4(3):99-107.

23. Reiber H, Lange P. Quantification of virus-specific antibodies in cerebrospinal fluid and serum: sensitive and specific detection of antibody synthesis in brain. Clin Chem. 1991;37(7):1153-1160.

24. Reiber H, Otto M, Trendelenburg C, Wormek A. Reporting cerebrospinal fluid data: knowledge base and interpretation software. Clin Chem Lab Med. 2001;39(4): 324-332.

25. Jarius S, Eichhorn P, Wildemann B, Wick M. Usefulness of antibody index assessment in cerebrospinal fluid from patients negative for total-IgG oligoclonal bands. Fluids Barriers CNS. 2012;9(1):14

26. Padilla-Docal B, Dorta-Contreras AJ, Bu-Coifiu-Fanego R, Rodríguez-Rey A, Gutiérrez-Hernández JC, de Paula-Almeida SO. Reibergram of intrathecal synthesis of C4 in patients with eosinophilic meningitis caused by angiostrongylus cantonensis. Am J Trop Med Hyg. 2010;82(6):1094-1098.

27. Reiber H. Knowledge-base for interpretation of cerebrospinal fluid data patterns: essentials in neurology and psychiatry. Arq Neuropsiquiatr. 2016;74(6):501-512.

28. Jarius S, Franciotta D, Paul F, et al. Cerebrospinal fluid antibodies to aquaporin-4 in neuromyelitis optica and related disorders: frequency, origin, and diagnostic relevance. J Neuroinflammation. 2010;7:52.

29. Jurynczyk M, Messina S, Woodhall MR, et al. Clinical presentation and prognosis in MOG-antibody disease: a UK study. Brain. 2017;140(12):3128-3138.

30. Jarius S, Pellkofer H, Siebert N, et al. Cerebrospinal fluid findings in patients with myelin oligodendrocyte glycoprotein (MOG) antibodies, part 1: results from 163 lumbar punctures in 100 adult patients. J Neuroinflammation. 2020;17(1):261.

31. Mariotto S, Ferrari S, Monaco S, et al. Clinical spectrum and IgG subclass analysis of anti-myelin oligodendrocyte glycoprotein antibody-associated syndromes: a multicenter study. J Neurol. 2017;264(12):2420-2430.

32. Bonnan M. Intrathecal IgG synthesis: a resistant and valuable target for future multiple sclerosis treatments. Mult Scler Int. 2015;2015:296184.

33. Akaishi T, Takahashi $\mathrm{T}$, Fujihara $\mathrm{K}$, et al. Impact of intrathecal IgG synthesis on neurological disability in patients with multiple sclerosis. Mult Scler Relat Disord. 2020; 45:102382.

34. Kowarik MC, Astling D, Gasperi C, et al. CNS aquaporin-4-specific B cells connect with multiple B-cell compartments in neuromyelitis optica spectrum disorder. Ann Clin Transl Neurol. 2017;4(6):369-380.

35. Höftberger R, Guo Y, Flanagan EP, et al. The pathology of central nervous system inflammatory demyelinating disease accompanying myelin oligodendrocyte glycoprotein autoantibody. Acta Neuropathol. 2020;139(5):875-892.

36. Ciotti JR, Eby NS, Wu GF, Naismith RT, Chahin S, Cross AH. Clinical and laboratory features distinguishing MOG antibody disease from multiple sclerosis and AQP4 antibody-positive neuromyelitis optica. Mult Scler Relat Disord. 2020; 45:102399.

37. Chihara N, Aranami $\mathrm{T}$, Oki S, et al. Plasmablasts as migratory IgG-producing cells in the pathogenesis of neuromyelitis optica. PLoS One. 2013;8(12):e83036.

38. Bennett JL, Lam C, Kalluri SR, et al. Intrathecal pathogenic anti-aquaporin-4 antibodies in early neuromyelitis optica. Ann Neurol. 2009;66(5):617-629.

39. Klawiter EC, Piccio L, Lyons JA, Mikesell R, O'Connor KC, Cross AH. Elevated intrathecal myelin oligodendrocyte glycoprotein antibodies in multiple sclerosis. Arch Neurol. 2010;67(9):1102-1108.

40. Jarius S, Ruprecht K, Kleiter I, et al. MOG-IgG in NMO and related disorders: a multicenter study of 50 patients, part 1: frequency, syndrome specificity, influence of disease activity, long-term course, association with AQP4-IgG, and origin. J Neuroinflammation. 2016;13(1):279.

41. Mariotto S, Gajofatto A, Batzu L, et al. Relevance of antibodies to myelin oligodendrocyte glycoprotein in CSF of seronegative cases. Neurology. 2019;93(20):e1867-e1872.

42. Aoe S, Kume K, Takata T, et al. Clinical significance of assaying anti-MOG antibody in cerebrospinal fluid in MOG-antibody-associated diseases: a case report. Mult Scler Relat Disord. 2019;28:165-166.

43. Peschl P, Bradl M, Höftberger R, Berger T, Reindl M. Myelin oligodendrocyte glycoprotein: deciphering a target in inflammatory demyelinating diseases. Front Immunol. 2017;8:529.

44. Hacohen Y, Absoud M, Deiva K, et al. Myelin oligodendrocyte glycoprotein antibodies are associated with a non-MS course in children. Neurol Neuroimmunol Neuroinflamm. 2015;2(2):e81. 
45. Brown D, Katsura T, Kawashima M, Verkman AS, Sabolic I. Cellular distribution of the aquaporins: a family of water channel proteins. Histochem Cell Biol. 1995;104(1): $1-9$.

46. Mobasheri A, Marples D, Young IS, Floyd RV, Moskaluk CA, Frigeri A. Distribution of the AQP4 water channel in normal human tissues: protein and tissue microarrays reveal expression in several new anatomical locations, including the prostate gland and seminal vesicles. Channels. 2007;1(1):29-38.
47. Sato DK, Callegaro D, de Haidar Jorge FM, et al. Cerebrospinal fluid aquaporin-4 antibody levels in neuromyelitis optica attacks. Ann Neurol. 2014;76(2):305-309.

48. Keizer RJ, Huitema AD, Schellens JH, Beijnen JH. Clinical pharmacokinetics of therapeutic monoclonal antibodies. Clin Pharmacokinet. 2010;49(8):493-507.

49. Wang B, Lau YY, Liang M, et al. Mechanistic modeling of antigen sink effect for mavrilimumab following intravenous administration in patients with rheumatoid arthritis. J Clin Pharmacol. 2012;52(8):1150-1161. 


\section{Neurology}

\section{Difference in the Source of Anti-AQP4-IgG and Anti-MOG-IgG Antibodies in CSF in Patients With Neuromyelitis Optica Spectrum Disorder}

Tetsuya Akaishi, Toshiyuki Takahashi, Tatsuro Misu, et al.

Neurology 2021;97;e1-e12 Published Online before print May 12, 2021

DOI 10.1212/WNL.0000000000012175

\section{This information is current as of May 12, 2021}

\section{Updated Information \& Services}

References

Citations

Subspecialty Collections

Permissions \& Licensing

Reprints including high resolution figures, can be found at: http://n.neurology.org/content/97/1/e1.full

This article cites 49 articles, 8 of which you can access for free at: http://n.neurology.org/content/97/1/e1.full\#ref-list-1

This article has been cited by 3 HighWire-hosted articles: http://n.neurology.org/content/97/1/e1.full\#\#otherarticles

This article, along with others on similar topics, appears in the following collection(s):

Acute disseminated encephalomyelitis

http://n.neurology.org/cgi/collection/acute_disseminated_encephalomy elitis

All Demyelinating disease (CNS)

http://n.neurology.org/cgi/collection/all_demyelinating_disease_cns Multiple sclerosis

http://n.neurology.org/cgi/collection/multiple_sclerosis

Optic neuritis; see Neuro-ophthalmology/Optic Nerve

http://n.neurology.org/cgi/collection/optic_neuritis

Information about reproducing this article in parts (figures,tables) or in its entirety can be found online at:

http://www.neurology.org/about/about_the_journal\#permissions

Information about ordering reprints can be found online:

http://n.neurology.org/subscribers/advertise

Neurology ${ }^{\circledR}$ is the official journal of the American Academy of Neurology. Published continuously since 1951, it is now a weekly with 48 issues per year. Copyright Copyright ( 2021 The Author(s). Published by Wolters Kluwer Health, Inc. on behalf of the American Academy of Neurology.. All rights reserved. Print ISSN: 0028-3878. Online ISSN: 1526-632X.

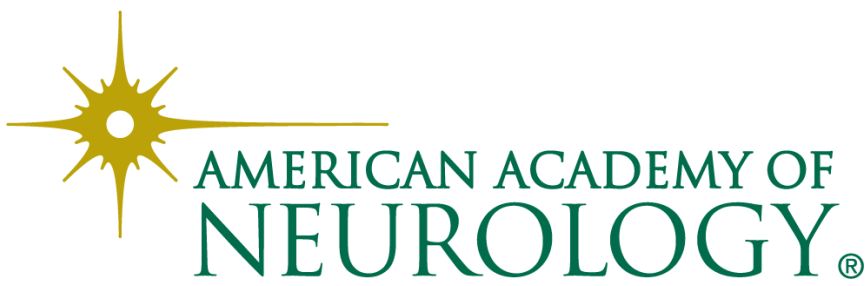

